\title{
Análisis de los marcos interpretativos de las políticas de infancia en Chile (2014-2018)*
}

\author{
María Pía Martin \\ Universidad de Chile. Facultad de Ciencias Físicas y Matemáticas. \\ Departamento de Ingeniería Industrial. Chile \\ mpmartin@dii.uchile.cl

\section{Joaquín Rozas Bugueño} \\ Universidad San Sebastián. Escuela de Trabajo Social. Chile \\ jirozas@uc.cl

\section{Jaime Alfaro Inzunza} \\ Universidad del Desarrollo. Facultad de Psicología. Chile \\ jalfaro@udd.cl
}

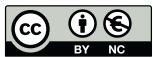

Recepción: 22-11-2018

Aceptación: 14-11-2019

Publicación: 01-07-2020

\section{Resumen}

El campo de las políticas hacia la infancia se muestra como un ámbito relevante a ser considerado en el análisis de políticas públicas. En él tienen un rol central los valores, ideologías y contextos situacionales que establecen la orientación simbólica de estas y el rol del Estado. Este artículo se propone indagar, a partir del análisis de marcos interpretativos, las tensiones que dificultaron la concreción de la reforma emblemática hacia la infancia y la adolescencia en Chile (2014-2018), con la identificación de los marcos interpretativos que se oponen a estos cambios o bien los impulsan. Desde la estrategia metodológica definida por la teoría fundamentada, se analizan 29 entrevistas semiestructuradas a actores relevantes y 42 discusiones parlamentarias. Los resultados establecen que hay dos marcos interpretativos en conflicto: el marco maestro de corte proteccionista con enfoque tutelar y el marco secundario de enfoque de derechos, donde se expresan las reformas, que busca sustituir al primero. A partir del análisis de alineamiento de marcos interpretativos, se establece que no se logra un cambio de paradigma. No obstante, se produce un cambio en las políticas mediante procesos de amplificación y extensión del marco interpretativo maestro. Se concluye que las dificultades de reformar las políticas hacia la niñez se concentran en el fuerte predominio de paradigmas y concepciones discrepantes con el ejercicio respecto, principalmente, del rol garante de derechos del Estado y la resistencia a considerar a los niños, las niñas y adolescentes como sujetos de derechos.

Palabras clave: análisis de política pública; infancia y adolescencia; teoría fundamentada; gobierno; significados; enfoque tutelar; enfoque de derechos

* Trabajo realizado en el marco del Proyecto Fondecyt 2016 No 11.160.330, «Cambio en las políticas hacia la infancia y adolescencia en Chile. Análisis de los marcos interpretativos de instituciones y actores relacionados». 


\section{Abstract. Analysis of child policy frames in Chile (2014-2018)}

Child policies are a relevant area of study in public policy analysis, where the values, ideologies and situational contexts that establish the symbolic orientation of such policies and the state play a central role. Based on the analysis of interpretive frameworks, this article aims to investigate the tensions that hindered the realization of the emblematic reform of the policy for children and adolescents in Chile (2014-2018) by identifying the interpretive frameworks that opposed or drove these changes. Following a grounded theory methodological approach, 29 semi-structured interviews of relevant actors and 42 parliamentary discussions are analyzed. The results reveal two conflicting interpretive frameworks: a protectionist master framework with a tutelary approach and a secondary framework with a rights-based approach, in which the reforms sought to replace the former approach. Through an alignment analysis of interpretive frameworks, it is established that a paradigm shift has not been achieved. However, a change in policies has occurred through amplification and extension processes of the master interpretive framework. It is concluded that the difficulties of reforming child policies are due to the strong predominance of paradigms and conceptions that mainly disagree with the state's role as a guarantor of rights and the resistance to consider children and adolescents as subjects of rights.

Keywords: public policy analysis; childhood and adolescence; grounded theory; government; meanings; tutelary approach; rights approach

\section{Sumario}

1. Antecedentes del análisis de marcos interpretativos de políticas

2. Antecedentes del análisis de políticas públicas de infancia y adolescencia: contexto y justificación de la reforma en Chile
3. Encuadre metodológico

4. Resultados

5. Conclusiones

Agradecimientos

Referencias bibliográficas

\section{Antecedentes del análisis de marcos interpretativos de políticas}

Con el fin de obtener una mejor comprensión de las respuestas de las políticas públicas a los problemas de la infancia y adolescencia, este artículo analiza los obstáculos en el proceso de reforma de las políticas públicas hacia los niños, las niñas y adolescentes. El estudio se realiza desde los modelos de análisis críticos e interpretativos (Yanow, 2000; Fischer, 2003), que permiten develar los marcos interpretativos implícitos y explícitos que contribuyen a explicar las resistencias, así como los impulsos al cambio de políticas (Fischer y Gottweis, 2012). Estos enfoques analíticos ponen énfasis en los marcos de ideas y en las prácticas discursivas institucionalizadas (Schmidt y Radaelli, 2004), donde están implicadas y adquieren relevancia dimensiones como significados, valores, creencias e ideología. Se opta específicamente por el análisis de frames (Goffman, 1986). Se utiliza el análisis de marcos interpretativos de políticas públicas, los cuales se entienden como principio organizativo que transforma 
la información fragmentada y circulante en un problema político estructurado y significativo, en el que se incluye implícita o explícitamente una solución (Verloo, 2005).

Los marcos interpretativos de las políticas son herederos del interaccionismo simbólico de Goffman, que se definine como:

(...) an interpretation scheme that structures the meaning of reality (...) an organizing principle that transforms fragmentary or incidental information into a structured and meaningful policy problem, in which a solution implicitly or explicitly enclosed. (Verloo, 2005: 19-20)

Asimismo, se entienden como construcciones sociales de modelos de realidad a partir de la elaboración de sentidos por parte de los actores de políticas (Hulst y Yanow, 2016; Verloo, 2005), que guían el entendimiento de la realidad y se encuentran en una situación de competencia y negociación constante. Cuando un marco interpretativo de política se impone sobre otros, estructura un discurso hegemónico excluyendo formas alternativas de entender un problema público y se posiciona como marco maestro (Broad y Turnbull, 2018; Barbieri, 2015; Bosomworth, 2015). Una consideración fundamental desde esta perspectiva analítica es que se entiende que la formulación de políticas no es un proceso de resolución de problemas de acuerdo con criterios racionales y técnicos (Schön y Rein, 1994), sino que es construida por los actores en base de sus marcos de interpretación. Los frames gobernarían el entendimiento de los problemas políticos y la construcción de los contenidos que guían estas percepciones y que responden a determinados contextos históricos, políticos y sociales, con la construcción en estos procesos de la política pública.

La potencialidad de este análisis se ha visto respaldada en la creciente importancia y el uso de marcos interpretativos en el estudio del proceso y las trayectorias de las políticas (Schön y Rein, 1994) en ámbitos como: las políticas de género (Verloo, 2005; Lombardo y Meier, 2009); las de migración (Roggeband, 2010); las políticas sociales (Steensland, 2008); las culturales (Barbieri, 2015); los movimientos sociales y la acción colectiva (Benford y Snow, 2000), entre otros, con la acumulación de un volumen creciente de evidencia empírica (Roberts, 2016; Shim et al., 2015).

Desde esta perspectiva, se entiende que las políticas públicas están atravesadas permanentemente por dinámicas de competencia entre actores, mediante ideas y argumentos para establecer cómo se define el problema público (Stone, 2002), y cuáles son las narrativas de políticas que promueven el consenso dentro de grupos de actores políticos. Lo que permite identificar cuáles son las diferencias entre los actores que llevan a definir el ellos y el nosotros, y distinguir de esta manera los espacios para los consensos que posibilitan la acción colectiva de los actores, a la vez que los procesos de conflicto (Roberts, 2016). Se recoge específicamente la reflexión de Hajer (2009), quien establece cómo las narrativas pueden acomodar tanto el consenso como el conflicto en arenas de deliberación de políticas. De manera que el análisis de los marcos interpre- 
tativos permite establecer cuáles son esos conflictos que obstaculizan la formulación de las reformas de políticas y cuáles son los consensos que las facilitan.

Asimismo, el análisis de marcos enfatiza la posición y disputas de estos actores respecto a la política pública recurriendo al análisis de las estructuras subyacentes de creencias, percepción y conceptualización. Más específicamente, se analizan las implicaciones de los issues de la política pública, en particular cuando hay controversias intratables, que son inmunes a la resolución por la apelación de los hechos, donde priman marcos interpretativos en conflicto sobre una situación o problema (Shim et al., 2015) respecto a los diagnósticos de lo que está mal y que requiere ser solucionado y cuáles son los hechos más relevantes (Schön y Rein, 1994). Es decir, cuando hay conflictos de significados, sentidos e interpretaciones aparentemente contradictorios que son estimados como verdaderos por quienes los conducen (Stone, 2002; Yanow, 2000). Los marcos interpretativos de las políticas constan de dos niveles: el primario, en el que define de forma abstracta el problema público; y el secundario, en el que las ideas se proyectan en un desarrollo operativo y construyen el diagnóstico y las soluciones del problema en cuestión (Bosomworth, 2015; Benford y Snow, 2000). El establecimiento abstracto del marco interpretativo se relaciona con el nivel más normativo de la definición del problema público y del porqué es relevante intervenir dicho problema (Bosomworth, 2015; Fisher, 2003). Según Rein y Schön (1996), este nivel se construye de acuerdo a lo que los actores de las políticas piensan normativamente del problema y cómo creen que debería ser la situación para resolverlo. Particularmente, en el primer nivel cobran relevancia los issues. En el nivel del diagnóstico (o foco-problema) y pronóstico (modelo de solución), los marcos interpretativos de las políticas establecen las causas y atribuciones de responsabilidad, y derivan además a una llamada a la acción asociada a soluciones para subsanar el problema público construido (Verloo, 2005; Benford y Snow, 2000). Al establecer los marcos interpretativos, se pueden determinar tanto el marco maestro, secundario y los conflictos de marcos (Steensland, 2008; Lombardo y Meier, 2009; Roberts, 2016; Shim et al., 2015), que obstaculizan o permiten acuerdos para el cambio de políticas públicas.

Los primeros análisis para explicar el cambio partieron del análisis de procesos de acción colectiva de movimientos sociales realizado por Snow y sus colaboradores (1986), quienes introdujeron el concepto de «alineamiento de marcos», referido a la capacidad de enlace de los marcos presentes en la acción colectiva, que posibilita la construcción de una interpretación compartida del problema y su solución. El concepto de alineamiento de marcos ha sido utilizado en el estudio y la comprensión del cambio de políticas públicas; respecto a las disputas simbólicas donde el marco secundario busca desplazar de la hegemonía al marco maestro, para generar el impulso al cambio, asociado a la legitimidad simbólica de un marco respecto a otro (Barbieri, 2015).

El alineamiento de marcos puede ser de cuatro tipos (Barbieri, 2015; Snow, et al., 1986): 1) frame bridging, donde se vinculan dos o más marcos coherentes entre sí, pero desconectados respecto a un asunto particular de política pública; 
2) frame amplification, en referencia a la integración de diversos marcos a través de un valor o creencia que permite una estrategia discursiva de difusión y legitimación; 3) frame extension, en referencia al alineamiento con el marco maestro que se produce a través de la reorganización de los valores de cada actor; 4) frame transformation, referido a la redefinición completa o parcial de un marco.

Barbieri (2015) plantea que el alineamiento de marcos tiene un efecto en el cambio de política cuando se alinean marcos específicos con el marco maestro, lo que conduce a la movilización y posibilita, de esta manera, analizar la capacidad de agencia de los actores en el cambio. Y permite explicar el cambio de políticas como resultado de la interacción simbólica entre actores políticos, dentro de un marco institucional que impone ciertas restricciones y posibilidades. Tanto la amplificación como la extensión del marco de políticas son procesos de alineamiento dinámicos que comportan la modificación de los propios marcos de políticas de los diferentes actores. En los procesos de alineamiento, los actores reorganizan sus propias valoraciones y reconstruyen diagnósticos, pronósticos y motivaciones para actuar en el proceso de políticas.

\section{Antecedentes del análisis de políticas públicas de infancia y adolescencia: contexto y justificación de la reforma en Chile}

Las políticas hacia los niños, las niñas y adolescentes son consideradas como parte de las políticas de protección social y, más específicamente, forman parte de los sistemas de bienestar (Gilbert et al., 2011), pero plantean un desafío particular a los enfoques tradicionales en el análisis del estado de bienestar, respecto a cómo abordan estos sistemas de protección los problemas específicos de la niñez y su relación con esta (Pösö et al., 2014).

Gilbert y colaboradores (2011), al analizar los sistemas de EE. UU., Canadá, Inglaterra, Suecia, Finlandia, Dinamarca, Noruega, Bélgica y Holanda, distinguen tres tipos de sistemas de protección social: 1) focalizado en el niño, donde el Estado tiene una función tutelar ya que promueve el cuidado familiar o la adopción, y existe un reemplazo de la familia por el Estado en los casos en que sea necesario, con el fin de proporcionar bienestar a los niños, las niñas y adolescentes; 2) servicio familiar, sistema en el que las políticas de Estado se centran en el fortalecimiento de las familias y sus vínculos sociales a partir del acompañamiento psicosocial; y 3) desarrollo infantil, caracterizado por la relevancia que el Estado confiere a los mecanismos legales individuales para la protección de la infancia, sistema que se convierte en un ente fiscalizador. Las propuestas de políticas públicas que generan mayor consenso son aquellas que fortalecen las capacidades de las familias (Duffy et al., 2018), y son las familias más pobres, como establece Harris (2014), las que están vinculadas a los servicios de bienestar para familias y niños y a la política social en general.

En este sentido, la discusión se relaciona con los sistemas de protección a la niñez y adolescencia, entendidos como sistemas de regulación legal y judicial que buscan determinar, investigar y proteger a los niños y niñas frente a los riesgos de abuso, maltrato, abandono, o incluso de perjudicarse a sí mismos por 
su comportamiento antisocial, y actúan a través de intervenciones específicas con o sin el consentimiento de los niños y las familias involucradas (Pösö et al., 2014). Dicho de otro modo, los sistemas de protección a los niños, las niñas y adolescentes integran directrices públicas, políticas sociales e intervenciones que tienen como objeto preferencial a quienes son vulnerados en sus derechos, pero también conforman políticas más amplias y universales dirigidas a este sector (Gilbert et al., 2011).

Tradicionalmente, los estudios de sociología y ciencias sociales en general han considerado a la niñez como inseparable conceptualmente de la familia, la mujer y los adultos demarcando una posición de menor jerarquía respecto a la adultez (Mayall, 2000). Desde los años 80 en adelante, se han desarrollado diversas perspectivas que han considerado a niños y niñas como sujetos sociales con agencia y como una unidad de análisis en sí misma (James, 2013; Gaitán, 2010; Mayall, 2000). Desde estas perspectivas, se concibe a la niñez como un constructo social, que varía histórica y culturalmente e incluye mandatos, reglas y pautas relacionadas con la forma en que un niño o una niña debe ser en determinada sociedad (Gómez-Mendoza y Alzate-Piedrahíta, 2014; James, 2013; Gaitán, 2010; Mayall, 2000). Con el planteamiento, en consecuencia, de que la niñez debe asumirse como fenómeno social y político anclada en un determinado contexto social (Gómez-Mendoza y Alzate-Piedrahíta, 2014) y de que es necesario reconocer la existencia de diferentes formas de entender la niñez.

Estudios desarrollados desde esta perspectiva establecen que los sistemas de protección desarrollados para la infancia están guiados por distintas perspectivas sobre cómo se entiende la niñez y la adolescencia (Pösö, et al., 2014; Gilbert et al., 2011), con la existencia de una relevante diversidad y tensión respecto a cuáles son los objetivos que guían a estos sistemas. Se identifican conflictos entre perspectivas liberales y proteccionistas, entre visiones Estadocéntricas y niño-céntricas, que implican diversas maneras de gestionar su protección (Gómez-Mendoza y Alzate-Piedrahíta, 2014; Villalta y Llobert, 2015; Mayall, 2000). Estas distintas visiones sobre los quehaceres institucionales y los sentidos, orientaciones y contenidos de la protección social se reflejan en los debates sobre el desarrollo de leyes, normativas, políticas públicas y sus cambios o reformas, así como en la emisión de directrices técnicas, y la distribución y montos presupuestarios (Collins, 2018). De esta manera, la formulación y la implementación de las políticas públicas sobre la niñez están atravesadas por la disputa y la negociación entre actores con distintas posturas sobre la realidad social y política (Villalta y Llobet, 2015), con diferentes concepciones sobre la equidad o la justicia como conceptos normativos que pueden ser siempre interpretados en diversas vías en conflicto (Stone, 2002).

En este contexto, los marcos analíticos y metodológicos desarrollados por el enfoque de análisis de marcos interpretativos de la política pública (Hulst y Yanow, 2016; Verloo, 2005) son una herramienta que permite develar y establecer cuáles son las aproximaciones valóricas y cognitivas respecto a los sistemas de protección de los niños, las niñas y adolescentes que dan fundamento a las reformas de políticas en este ámbito. 
Siguiendo este enfoque analítico, este trabajo busca contribuir al vacío de conocimientos sobre las ideas, valores y creencias en base a las cuales los actores implicados en las políticas públicas hacia los niños, las niñas y adolescentes elaboran diagnósticos, generan soluciones y establecen responsabilidades sobre esa situación o problema, así como las intervenciones que son posibles o aceptables de proyectar. Específicamente, se busca analizar de qué manera los marcos interpretativos de las políticas que conducen los actores e instituciones de la política pública a la niñez en Chile condicionan, obstaculizan o potencian cambios en la formulación de una nueva política pública que responda a los problemas públicos en materia de infancia y adolescencia.

En Chile, pese a la ratificación de la Convención de Derechos del Niño (CDN) en 1990, no hay un órgano rector ni una normativa que exprese los principios de la convención (Morlachetti, 2013). Existe heterogeneidad de la oferta programática (Larraín, 2011) y una preeminencia de la intervención judicial (Vergara del Solar, 2014), y se formulan las políticas desde un modelo tutelar (Grau, 2011; Ortiz, 2009), donde la noción de niñez es receptora de las acciones asistencialistas del Estado y como titular de derechos (Herrera y Aravena, 2015). En el enfoque tutelar, se establece que, cuando las familias fallan, el Estado debe intervenir y, como medida extrema, separar al niño de su familia y internarlo en organismos públicos dependientes de un servicio público específico, el Servicio Nacional de Menores (Sename), dependiente del Ministerio de Justicia y Derechos Humanos. Bajo este paradigma, el juez adquiere un rol central, ya que es el encargado de disponer las medidas que deben aplicarse a los niños en situación irregular y determinar las intervenciones o sanciones para cada caso particular.

Esto se produce, aunque la CDN provoque una ruptura con el paradigma tutelar, a partir de la preeminencia del enfoque de derechos en las políticas hacia la niñez (Sepúlveda, 2014; Ortiz, 2009), con nuevos derechos de ciudadanía para los niños, las niñas y adolescentes que los consagran como sujetos legales independientes con derecho a participación (Earls, 2011). Los derechos establecidos en la $\mathrm{CDN}$ son sociales, transindividuales, atribuidos a una categoría social, y su garantía es esencialmente política. Tienen que ser garantizados por toda la sociedad, ya que son derechos que una generación busca fundar en una nueva generación emergente como parte de un proceso de emancipación social (Bustelo, 2005).

El programa de gobierno de Bachelet (2014-2018), ${ }^{1}$ al buscar la integración del paradigma de enfoque de derechos, reformó las políticas públicas de niñez

1. Se refiere al segundo mandato de Bachelet, de 2014 a 2018, apoyado por la coalición de centro-izquierda Nueva Mayoría, conformada por el Partido Socialista (PS), el Partido por la Democracia (PPD), el partido Demócrata Cristiano (DC), el Partido Radical (PR), el Partido Comunista (PC), Izquierda Ciudadana (IC) y MAS Región. El primer mandato de Bachelet fue entre 2006 y 2010, apoyado por la coalición de centro-izquierda Concertación de Partidos por la Democracia, sin el PC. Cabe mencionar que el sistema de partidos chileno se ordena en un continuo izquierda-derecha, donde los partidos con representación parlamentaria de izquierda a derecha para el período 2014-2018 eran Movimiento Autonomista (MA), Revolución Democrática (RD), PC, IC, MAS Región, PS, PPD, PR, DC, Evópoli, Renovación Nacional (RN) y Unión Demócrata Independiente (UDI). 
con el objetivo de generar un sistema de protección integral, con el Estado como garante de los derechos establecidos en la convención. Ello implicaba un cambio radical, ya que las políticas públicas del país se caracterizan por ser sectorizadas y focalizadas, sin un sistema coordinado, y existen múltiples ministerios, servicios y organizaciones sociales encargados de la protección social a la niñez ${ }^{2}$ (Bedregal et al., 2014; Maldonado, 2014). Una de las primeras medidas al iniciar el gobierno fue la creación del Consejo Nacional de la Infancia, ${ }^{3}$ organismo temporal instaurado para apoyar e impulsar la formulación de la reforma, con la coordinación de las acciones de los distintos actores políticos involucrados. La reforma contempló el envío al Congreso de cinco proyectos de ley centrales de un paquete presupuestado de dieciséis proyectos, que buscaban la creación de un sistema integral con enfoque de derechos para la niñez.

La reforma estuvo centrada en cambios normativos ${ }^{4}$ que se sostenían en el enfoque de derechos y en la instalación de un Sistema de Garantías de Derechos de la Niñez, integrado por el conjunto articulado de políticas, instituciones y normas cuyo objetivo era velar por la protección integral y el ejercicio de los derechos de los niños, las niñas y adolescentes establecidos en la convención, con la articulación de la oferta pública para asegurar el pleno desarrollo de todos ellos. ${ }^{5}$ Este proyecto generó amplia controversia y no logró ser aprobado.

Por otra parte, otra batería de proyectos implicaba fortalecer la arquitectura institucional con foco en la niñez, con la inclusión de la creación de la Defensoría de la Niñez y la Subsecretaría de la Niñez en el Ministerio de Desarrollo Social, ambos proyectos aprobados y actualmente operativos y en funcionamiento. Además, se buscó separar administrativamente los servicios centrados en infractores de ley de los de protección a la infancia vulnerada en sus derechos, que actualmente están en manos de un mismo servicio, el Servicio Nacional de Menores (Sename). Con ese fin, se enviaron los proyectos que creaban el Servicio Nacional de Reinserción y el Servicio Nacional de

2. El sector de niñez y adolescencia está conformado por los ministerios de Educación, Salud, Desarrollo Social (MDS) y Justicia y Derechos Humanos, el Servicio Nacional de Menores (Sename), 198 programas anidados en distintos ministerios y más de 400 organizaciones de la sociedad civil que prestan atención en residencias y centros abiertos, y que son financiadas por Sename mediante licitación de proyectos en las líneas de residencia, protección y adopción.

3. Creado el 14 de marzo de 2014, a una semana de asumir la presidencia Bachelet (11 de marzo de 2014), mediante decreto presidencial. El consejo cesó sus funciones el 1 de abril de 2018, a un mes de asumir el nuevo gobierno Sebastián Piñera (2018-2022), de centroderecha.

4. La reforma a la política de niñez y adolescencia estuvo conformada por cinco proyectos de ley: creación de la Subsecretaría de la Niñez, instauración de la Defensoría de la Niñez; separación del Servicio Nacional de Menores (Sename) en el Servicio Nacional de Protección Especializada (Boletín 11.176-07) y el Servicio Nacional de Reinserción (Boletín 11.174-07), y la conformación del Sistema de Garantías de Derechos de la Niñez (Boletín 10.315-18). De esta batería de proyectos solo se aprobaron los dos primeros - Ley 21.090 y Ley 21.067, respectivamente.

5. Definición del Sistema de Garantías de Derechos de la Niñez establecida en el proyecto de ley (Boletín 10.315-18), aún en discusión en el Congreso chileno. 
Protección Especializada, los cuales tampoco lograron aprobarse y están aún en discusión en el Congreso. De manera que solo dos de los cinco proyectos centrales del paquete de iniciativas legales de la reforma se constituyeron en ley vigente actualmente.

El resto de los proyectos que eran parte del paquete de dieciséis modificaciones que estaba planificado enviar al Congreso para su discusión y que contemplaban cambios en el sistema de financiamiento, fiscalización y regulación de las organizaciones de la sociedad civil que proveen servicios de protección, así como la reforma a los procedimientos judiciales, a la Ley de Menores (que data de 1967), entre otras, nunca llegaron a enviarse.

Es en el marco de este proceso de cambio de políticas que este trabajo, desde un análisis de marcos interpretativos de las políticas, busca determinar los factores que contribuyen a explicar los obstáculos y las dificultades para la aprobación de una ambiciosa reforma que buscaba encarnar los principios de la Convención de Derechos del Niño bajo el paradigma del enfoque de derechos.

\section{Encuadre metodológico}

El encuadre metodológico de esta investigación está vinculado a los enfoques interpretativos de análisis de políticas (Fischer, 2003; Yanow, 2000; Huslt y Yanow, 2016), tal como ya se ha expuesto.

Operacionalmente, este trabajo analizó los marcos interpretativos de las políticas que estuvieron presentes en el proceso de reforma de la política de niñez y adolescencia en Chile (en el período de gobierno de Bachelet de 2014 a 2018).

Se analiza específicamente el problema de la política pública, que estructura un diagnóstico con distintas representaciones tanto implícitas como explícitas, así como prejuicios (entendidos como filtros construidos social y culturalmente) a través de los cuales se percibe e identifica un problema y las causas que lo originan, y un pronóstico con las posibles soluciones, las acciones que se deben emprender y quienes son los que deben solucionarlo.

Asimismo, se analizan los marcos sociales, culturales e históricos específicos, donde se establecen atribuciones de roles en función de ese contexto. Específicamente, el marco maestro expresa la estructura discursiva predominante, y en un contexto donde hay situaciones de poder diferenciadas, donde los discursos tienen un valor ponderado según quien los emita. De esta manera, hay quienes tienen una voz dominante en el debate político, mientras que otros son excluidos de él. El lenguaje y el discurso son analizados como herramienta estratégica para perseguir determinados fines, crear y manipular situaciones o provocar representaciones en disputa en un mismo acontecimiento. Así, también se analizan los marcos secundarios que pueden estar en conflicto o bien en equilibrio con el marco dominante, donde se incorporan visiones distintas y/o en competencia sobre el problema y su solución. Conjuntamente se analiza la alineación de marcos que permite el cambio de políticas, entendido como un proceso en el que los actores revalorizan sus propios puntos de vista y recons- 
truyen diagnósticos, pronósticos y motivaciones para actuar en un ámbito de política pública (Barbieri, 2015; Snow et al., 1986).

Para el análisis del cuerpo empírico de datos, se utilizó la teoría fundamentada, Grounded Theory (Strauss y Corbin, 2002), que permite el análisis descriptivo y relacional (codificación abierta, codificación axial y codificación selectiva) y la tipificación de las dinámicas implicadas y de los procesos relacionales de los que forman parte. En la codificación abierta se clasifica la información mediante códigos y se establecen categorías, subcategorías y propiedades. La codificación axial es el proceso de relacionar códigos (categorías y propiedades). Finalmente, la codificación selectiva es la relación conceptual y teórica que integra en una categoría central las relaciones establecidas en la codificación axial. El análisis se realizó con el software N-vivo 11.

La muestra estuvo constituida por 29 entrevistas semiestructuradas a actores relevantes de gobierno (ver cuadro 1), organizaciones internacionales y organizaciones de la sociedad civil, además del análisis de 42 discusiones parlamentarias $^{6}$ de los proyectos de ley, mociones parlamentarias, leyes y convenios internacionales en niñez (incluyendo el trabajo de comisiones) introducidos e impulsados por las reformas del gobierno de Bachelet (2014-2018). Para la

Cuadro 1. Caracterización de entrevistados.

\begin{tabular}{|c|c|c|}
\hline Ámbito & Entrevistados (pertenencia y número) & Policy frame adscrito \\
\hline \multirow[t]{3}{*}{$\begin{array}{l}\text { Gobierno } \\
16 \text { entrevistas }\end{array}$} & $\begin{array}{l}\text { Asesores ministerios: Presidencia (2), Desarrollo } \\
\text { Social (4), Consejo de la Infancia (2) }\end{array}$ & Enfoque derechos \\
\hline & Educación (1) & \\
\hline & $\begin{array}{l}\text { Representantes Ministerio de Hacienda (2), de Salud } \\
\text { (1), Justicia (3), directora Servicio Nacional del Menor, } \\
\text { Sename (1) }\end{array}$ & Proteccionista \\
\hline \multirow[t]{2}{*}{$\begin{array}{l}\text { ONG } \\
6 \text { entrevistas }\end{array}$} & $\begin{array}{l}\text { Representantes de organizaciones de atención } \\
\text { a la niñez, reconocidos por Sename (3) }\end{array}$ & Proteccionista \\
\hline & $\begin{array}{l}\text { Representantes de organizaciones especializadas } \\
\text { en niñez (3) }\end{array}$ & Enfoque derechos \\
\hline $\begin{array}{l}\text { Nivel local } \\
1 \text { entrevista }\end{array}$ & Encargado de redes municipales (1) & Enfoque derechos \\
\hline \multirow{2}{*}{$\begin{array}{l}\text { Organismos } \\
\text { Internacionales } \\
3 \text { entrevistas }\end{array}$} & Oficial Banco Mundial (1) y oficial BID (1) & Proteccionista \\
\hline & Oficial OIT (1) & Enfoque derechos \\
\hline \multirow{2}{*}{$\begin{array}{l}\text { Académico } \\
3 \text { entrevistas }\end{array}$} & Académico especialista en infancia (2) & Frame proteccionista \\
\hline & Académico especialista en infancia (1) & Enfoque derechos \\
\hline
\end{tabular}

Fuente: elaboración propia.

6. El análisis de discusiones parlamentarias permite indagar de qué forma los actores movilizan los marcos interpretativos en la elaboración de política pública. 
realización de entrevistas, se siguieron los resguardos éticos, con solicitudes de autorización y consentimientos informados para llevar a cabo cada entrevista, y con el respeto a la privacidad y a los tiempos de quienes participaron del proceso de investigación.

A partir del análisis de la discusión parlamentaria, se organizó la información sobre actores presentes en la discusión y la trayectoria de los proyectos de ley. Tanto las entrevistas como los proyectos de ley fueron procesados según las categorías establecidas y en función de los objetivos y el marco teórico sobre marcos interpretativos de las políticas, de manera que se establecieron elementos emergentes relevantes para la investigación. El proceso siguió la teoría fundamentada (Conlon et al., 2015; Strauss y Corbin, 2002), lo que propició la creación de categorías abiertas de forma exploratoria para luego establecer códigos relacionales o axiales con el fin de ir refinando el análisis. Finalmente, se realizó una tercera codificación (selectiva), que permitió integrar las relaciones establecidas en las codificaciones axiales para discernir, comprender y dar sentido a los datos.

\section{Resultados}

\subsection{Marcos interpretativos en la política de niñez en Chile (2014-2018)}

Los resultados muestran que en la política chilena de niñez y adolescencia existe un marco interpretativo maestro proteccionista que define como problema público principal la falta de focalización de la acción del Estado en la protección de los niños, las niñas y adolescentes que se encuentran en estado de vulneración de derechos o situación irregular. Este marco concibe a los niños, las niñas y adolescentes como individuos en formación e incompletos, por lo que el Estado debe otorgarles protección en caso de que la familia o adultos responsables no estén en condiciones de garantizar sus derechos, lo que está en concordancia con el enfoque de focalización en infancia vulnerada, generalmente de contextos de pobreza.

La fragilidad y vulnerabilidad del niño nos llevan a asumir el máximo de compromisos y de medidas para su defensa. Hoy es prioritario el interés superior del niño, de la niña y del adolescente, por lo que debemos prevenir su abandono y su indefensión, así como asumir su representación. (Cámara de Diputados [PDC, centroizquierda]. Boletín 10.584, 04/10/2017)

Se establece otro marco interpretativo secundario de enfoque de derechos que concibe como problema principal la falta de una arquitectura normativa e institucional en que el Estado sea garante de los derechos establecidos en la convención. Los niños, las niñas y adolescentes son considerados sujetos de derechos con autonomía progresiva — con independencia creciente respecto a sus padres o tutores, lo que está supeditado a la dependencia biológicabiográfica de los niños, las niñas y adolescentes - y derecho a ser escuchados. 
En este sentido, este frame está más alineado normativamente con los estándares de la convención y tendría consistencia con el sistema de garantías de derechos de la niñez.

(...) aquí lo que tenemos que apoyar es el objetivo final, y el objetivo final es el desarrollo integral de los niños, de eso no hay discusión; y el objetivo final no es cumplir con la Convención, sino que el objetivo final es el desarrollo integral de los niños, ¿no? (Entrevista a un profesional del Banco Mundial, 26/5/2017)

El marco interpretativo proteccionista tiene un diagnóstico que considera que: 1) la intervención en la niñez y la adolescencia vulnerada o en situación irregular no está siendo efectiva; 2) se deben priorizar en la niñez vulnerada en sus derechos; 3) la familia debe ser prioritaria.

Cómo somos capaces, como Estado, de responder a niños que hoy están absolutamente abandonados, a niños vulnerables de los cuales no nos hacemos cargo, a niños que son víctimas de violencia, de abandono o de discriminación, o que, en muchos casos, después de ser abandonados por la sociedad, terminan siendo infractores de ley. (Diputado Chahín [PDC, centroizquierda]. Boletín $10.315-18,12 / 04 / 2017)$

Desde el marco interpretativo maestro, más que una reforma, se requiere mejorar la efectividad de lo que se realiza. Es decir, habría que mantener el statu quo y mejorar la coordinación entre instituciones como una de las principales causas de la ineficiencia en la intervención:

(...) efectivamente las políticas, prestaciones y servicios se abordan con ópticas diferentes, según el énfasis sectorial, en cada caso (...) ello genera incoherencias en las políticas públicas, pues se produce duplicación o superposición de prestaciones y servicios para atender fenómenos similares (...). (Senador Alberto Espina. Trámite constitucional de la Ley 21.090 [RN, derecha], 12/7/2017)

Otra causa de las ineficiencias es el exceso de burocracia. Bajo la construcción de este marco, el aumento de tamaño del Estado iría en detrimento del avance en el resguardo de los derechos de la niñez en situación de vulnerabilidad e infractores de ley, ya que los recursos se dispersarían, como se ejemplifica a continuación:

¿No hubiese sido preferible, en vez de seguir creando una institucionalidad de papel, sin atribuciones ni presupuesto suficiente para proteger en serio a nuestros niños, haber destinado esos mismos esfuerzos y recursos en, primero, dar solución al drama que viven día a día los niños vulnerados e infractores de ley de nuestro país? (Diputado Nicolás Monckeberg. Tramitación de la Defensoría de la Niñez [RN, derecha], 10/10/2017)

Respecto al rol que tiene la familia en el sistema de protección, los actores que se posicionan en este marco sostienen que el actual sistema ha fallado al 
no fortalecer las capacidades de la familia para proteger a los niños, las niñas y adolescentes. En este sentido, este aspecto del diagnóstico tiene similitud con la opción del sistema de servicio familiar, puesto que se prioriza el fortalecimiento de las habilidades de la familia para criar, educar y cuidar a sus hijos como estrategia tanto para prevenir vulneraciones como para reducir la intervención del Estado en las familias.

(...) la tendencia por centrar menos esfuerzo en la oferta institucional de programas y servicios de atención a familias para fortalecimiento y capacidad parental, en beneficio de formar directamente capacidades de cuidados y crianza en padres, madres o adultos... Luego si alguien está francamente desorientado, siente que se le va de las manos el tema de la disciplina, la norma, el límite, ‘̇a quién recurre? (Entrevista a un profesional del BID, 22/8/2017)

En consecuencia, quienes se posicionan desde el marco proteccionista apelan a la consideración y al resguardo del derecho preferente de los padres a educar a sus hijos, lo que prima ante cualquier autoridad o ante el Estado. De manera que el Estado puede promover la participación de los niños en el ámbito de su interés, pero son los padres quienes deciden sobre su educación, guía y orientación, y los que consideran su edad y su madurez. El Estado solo puede intervenir cuando los padres vulneran derechos fundamentales de sus hijos: «La obligación prioritaria de la familia es preocuparse de los hijos, y el Estado no puede interferir en esto» (senador Hernán Larraín, trámite constitucional de la Ley 20.090 [UDI, derecha], 15/3/2017).

En este frame proteccionista, la prioridad de las políticas públicas debe estar en los niños, las niñas y adolescentes que no cuentan con garantías para sus derechos, donde la familia no es capaz de brindar protección, y solo en esos casos de violaciones graves de derechos es el Estado el que debe reemplazar el rol primario que debe cumplir la familia.

Creemos que hoy la primera prioridad debiera ser hacerse cargo de la situación dramática (...) que están sufriendo niños que están siendo vulnerados en sus derechos todos los días ¡De esos niños debiéramos hacernos cargo hoy! (Senadora Ena von Baer. Tramitación de la Subsecretaría de la Niñez [UDI, derecha], 15/3/2017)

La responsabilidad del problema público se atribuye a familias en condición de pobreza, con mayor riesgo social. De manera que son las familias y los niños pobres el objeto de la política social en general. La acción del Estado debería focalizarse en ellos.

Como Estado se debe entender que el desarrollo y futuro de un país se basan en la forma en que se trata a todos los niños, sobre todo a los más vulnerables, a aquellos que nacen en las condiciones más difíciles. Aquello marca la diferencia en su futuro y desarrollo. (Diputado Robles. Tramitación del proyecto de ley de Defensoría de la Niñez [PRSD, centroizquierda], 10/10/ 2017) 
Respecto al marco de soluciones, el marco interpretativo maestro de tipo proteccionista está asociado a medidas de gestión que aumenten la eficiencia de la protección especializada, con la prioridad de aumentar la coordinación entre instituciones interventoras. También se requiere un cambio institucional creando dos nuevos servicios especializados: uno abocado a los niños, las niñas y adolescentes vulnerados en sus derechos y otro de reinserción de los adolescentes infractores de ley:

Lo más apremiante era la separación del Sename en una institucionalidad preocupada, por un lado, por los niños vulnerados en sus derechos y, por el otro, por los niños infractores de ley. Si a mí me hubiesen preguntado por dónde empezar, probablemente habría contestado que por la separación del Servicio en dos instituciones, porque es ahí donde tenemos la crisis más relevante, la que más le duele al alma de nuestro país en este momento. (Senadora Ena von Baer. Tramitación de la Subsecretaría de la Niñez [UDI-derecha], 16/01/2018)

El marco interpretativo secundario de enfoque de derechos es impulsado en la reforma y basa su diagnóstico de problema público en tres dimensiones: 1) ausencia de un sistema integral para la protección de la niñez; 2) necesidad de superar la focalización y avanzar hacia la provisión universal de derechos; 3) mejoramiento de la institucionalidad.

Se precisa una mirada distinta en nuestro país acerca de la niñez, no solo de la vulnerada (por cierto, esto nos duele en el alma); no solo de cómo se cambian los paradigmas para garantizar la protección del Estado y la sociedad toda, sino también para ver cómo promovemos políticas para que todos los niños y las niñas de Chile tengan garantizados sus derechos. (Senado [PS, izquierda]. Boletín 10.314-06, 21/07/2017)

La falta de un sistema de protección integral que haga efectivo el ejercicio estatal de garantía de derechos impediría tener una acción pública coordinada entre las organizaciones públicas y privadas que implementan las políticas públicas hacia ese sector:

(...) la acción del Estado en materia de infancia es precaria y fraccionada, sin ninguna articulación intersectorial (...) dicha situación, a estas alturas, es insostenible y da cuenta de la urgencia de poder contar con políticas universales de promoción y protección de los niños, niñas y adolescentes, y de un ente rector que las articule y las coordine. (Camila de la Maza, Corporación Opción. ${ }^{7}$ Tramitación de la Subsecretaría de la Niñez, 20/11/2017)

Respecto al problema de focalización de la política, se establece una segmentación en el entendimiento del problema, y se asocian las políticas

7. Corporación Opción es una de las instituciones privadas sin fines de lucro más grandes del país que es subsidiada por el estado. Presta servicios a niños, niñas y adolescentes vulnerados en sus derechos y con medidas de protección judicial. 
públicas hacia la niñez solo a quienes viven condiciones de vulnerabilidad por pobreza.

(...) la política pública [de niñez y adolescencia], a pesar de los avances que ha experimentado en distintas áreas, ha estado enfocada hacia la vulnerabilidad (...) se habla mucho de integralidad e intersectorialidad, pero a lo más se llega a la coordinación y a una mirada segmentada del desarrollo de las personas. (María Estela Ortiz, secretaria ejecutiva del Consejo de la Infancia. Tramitación de la Subsecretaría de la Niñez, 12/7/2016)

Mejorar la institucionalidad se relaciona con el hecho que se mantiene un sistema tutelar, en el cual las instituciones del sistema de justicia son las que deciden sobre las medidas de protección a niños, niñas y adolescentes, lo que refuerza la focalización del sistema en la vulneración de derechos e infractores de ley, en base a una institucionalidad reactiva y que segmenta a su población objetivo de acuerdo a sus carencias sociales.

(...) me parece que es contraintuitivo que todos los casos de vulneración, cualquiera, hoy estén radicados en la solución de los tribunales de familia. Si yo soy una madre que tengo que trabajar porque la vida me pone en esa situación y, probablemente, también por el tipo de educación que uno recibió se puede sentir culpable, abandonadora, etcétera, aunque adore y quiera a mi hijo... Y eso es lo que pasa hoy día, los jueces además resuelven desde sus experiencias vitales. Y sus experiencias vitales, te puedo asegurar, no tienen nada que ver con la señora que tiene que viajar al día seis horas para trabajar, que es dueña de casa o jefa de hogar sola. (Entrevista a la directora del Sename, 13/6/2017)

Estas tres dimensiones del diagnóstico del marco interpretativo de enfoque de derechos tienen por resultado la imposibilidad de avanzar hacia un sistema que garantice la autonomía progresiva de los niños, las niñas y adolescentes, pues no se resguarda el cumplimiento del rol garante del Estado bajo los estándares que fija la convención. Al establecerse la preeminencia de enfoques asistencialistas y tutelares del Estado, las acciones públicas son focalizadas en la población más pobre y en quienes han visto vulnerados sus derechos, sin que se establezcan políticas y programas universales que velen por los derechos de todos los niños, niñas y adolescentes. Ello conlleva, según señalan los actores de las políticas, que no se garanticen los derechos que la convención establece.

Constantemente hemos recibido observaciones al respecto [institucionalidad de protección a la niñez y adolescencia] (...) por nuestra falta de legislación y nuestra incapacidad para dictarla de una manera más efectiva, a fin de que sea posible aseverar que los derechos de los menores son protegidos como corresponde. (Senadora Isabel Allende. Discusión de trámite constitucional [PS, izquierda], 9/5/2017)

Los actores que sostienen este marco atribuyen la responsabilidad del problema a la falta de normas, políticas e instituciones del actual sistema de protec- 
ción, que no ha podido brindar una protección integral ni garantizar los derechos establecidos en la convención ni tener como eje el enfoque de derechos para asegurar la autonomía progresiva de los niños, las niñas y adolescentes:

(...) actualmente la institucionalidad existente se basa en el paradigma de la situación irregular de los niños y la doctrina tutelar, lo que ha generado que los problemas de los niños se tiendan a explicar a partir de las carencias individuales de los afectados, de tal manera que en la práctica se trata de un sistema de protección de menores altamente judicializado, en que la administración [del Estado] solo cumple un rol de prestador de servicios. (Mensaje del proyecto de ley de la Subsecretaría de la Niñez. Tramitación, 12/7/2016).

Finalmente, este marco interpretativo tiene como modelo de solución generar un cambio en el marco institucional que comporte nuevos organismos, leyes marco y políticas acordes al mandato de la convención, donde exista un armazón integral de actores que puedan ejecutar acciones intersectoriales y coordinadas que garanticen el goce de los derechos y la autonomía progresiva de los niños, las niñas y adolescentes. Ello implica un cambio legal para instaurar un sistema de garantías de derechos de la niñez con una mirada a largo plazo y el establecimiento de una institución que ejerza la rectoría del sistema de protección integral, como podría ser la Subsecretaría de la Niñez:

(...) se trata de tener cierta anticipación a los problemas, llegar a las regiones y tener mejores desplazamientos, que es algo en lo que el Ministerio de Desarrollo Social tiene mayor experiencia y organización. (Senador Bianchi. Discusión del trámite constitucional de la Ley 21.090 [independiente-derecha], 7/3/2017).

El marco interpretativo de las políticas se establece en el siguiente cuadro, que incluye las dimensiones señaladas:

El conflicto de marcos interpretativos, entre el marco maestro y el secundario, se expresa en el cuadro 2, donde se aprecian las diferencias respecto al foco-problema, la atribución de responsabilidad y el modelo de solución, que resume la discusión sobre la reforma impulsada y sus focos de resistencia y acuerdo.

Se desarrollan las divergencias que surgen entre los diferentes marcos interpretativos identificados. Estos conflictos se producen porque los marcos entran en competencia, ya que oponen sus concepciones tanto por la definición del problema público como por la solución del mismo (Bustelo y Lombardo, 2006 Lombardo, 2005; López, 2011).

El conflicto de marcos interpretativos de las políticas se estableció en torno a tres temas principales: el rol del Estado subsidiario vs. el rol del Estado garante de derechos; la autonomía progresiva de niños, niñas y adolescentes vs. la primacía del derecho de los padres a educar, guiar y orientar a sus hijos como límite frente a la acción del Estado, es decir, el derecho preferente no es del niño sino de los padres frente al Estado; y la focalización en la niñez vulnerable 
Cuadro 2. Conflicto de marcos interpretativos: proteccionista y enfoque de derechos en la política de niñez en Chile (2014-2018).

\begin{tabular}{|c|c|c|}
\hline Dimensión & Marco interpretativo proteccionista & $\begin{array}{l}\text { Marco interpretativo de enfoque } \\
\text { de derechos }\end{array}$ \\
\hline \multirow[t]{3}{*}{$\begin{array}{l}\text { Diagnóstico o } \\
\text { foco-problema }\end{array}$} & $\begin{array}{l}\text { Ineficiencia en la intervención a } \\
\text { la niñez vulnerada o en situación } \\
\text { irregular a causa de la excesiva } \\
\text { burocracia y la falta de coordinación } \\
\text { entre instituciones }\end{array}$ & $\begin{array}{l}\text { No existe un sistema integral entre los } \\
\text { distintos actores públicos y privados } \\
\text { que integran, bajo enfoque de dere- } \\
\text { chos, las políticas y los programas de } \\
\text { protección a niños, niñas y adoles- } \\
\text { centes }\end{array}$ \\
\hline & $\begin{array}{l}\text { No se le ha otorgado a la familia } \\
\text { la centralidad que le corresponde en } \\
\text { el sistema de protección a la niñez }\end{array}$ & $\begin{array}{l}\text { Mantenimiento del enfoque tutelar con } \\
\text { preeminencia de la judicialización de la } \\
\text { política de niños, niñas y adolescentes }\end{array}$ \\
\hline & $\begin{array}{l}\text { Los niños, las niñas y adolescentes } \\
\text { vulnerados no son prioridad política }\end{array}$ & $\begin{array}{l}\text { La intervención es asistencialista y } \\
\text { centrada en la niñez vulnerada y no } \\
\text { universal según derechos }\end{array}$ \\
\hline $\begin{array}{l}\text { Atribución de } \\
\text { responsabilidad }\end{array}$ & Pobreza vinculada a riesgo social & $\begin{array}{l}\text { Normas, instituciones y políticas no } \\
\text { acordes a la convención }\end{array}$ \\
\hline \multirow[t]{4}{*}{ Voz } & \multirow{2}{*}{$\begin{array}{l}\text { Actores del sector público, organiza- } \\
\text { ciones de la sociedad civil, académi- } \\
\text { cos, organismos internacionales } \\
\text { y partidos políticos. Los niños y las } \\
\text { niñas no tienen espacio de participa- } \\
\text { ción en la discusión pública. }\end{array}$} & $\begin{array}{l}\text { Actores del sector público, organiza- } \\
\text { ciones, sociedad civil, académicos, } \\
\text { organismos internacionales y partidos } \\
\text { políticos. }\end{array}$ \\
\hline & & $\begin{array}{l}\text { Niños y niñas participan en consulta } \\
\text { para la política nacional }\end{array}$ \\
\hline & $\begin{array}{l}\text { Partidos políticos de derechas } \\
\text { (Partido Unión Demócrata Indepen- } \\
\text { diente, Partido Renovación Nacional } \\
\text { y Partido Amplitud). }\end{array}$ & $\begin{array}{l}\text { Partidos políticos de izquierda (Partido } \\
\text { Comunista, Partido Socialista, Izquier- } \\
\text { da Ciudadana y Revolución Democrá- } \\
\text { tica). }\end{array}$ \\
\hline & $\begin{array}{l}\text { Partidos de centroizquierda como } \\
\text { Partido Demócrata Cristiano y Partido } \\
\text { por la Democracia, de la coalición } \\
\text { de centroizquierda gobernante de } \\
\text { Bachelet, Nueva Mayoría }\end{array}$ & $\begin{array}{l}\text { Partido Demócrata Cristiano y Partido } \\
\text { por la Democracia, ambos de la coali- } \\
\text { ción de centroizquierda gobernante de } \\
\text { Bachelet, Nueva Mayoría. }\end{array}$ \\
\hline $\begin{array}{l}\text { Pronóstico } \\
\text { o modelo de } \\
\text { solución }\end{array}$ & $\begin{array}{l}\text { Mejorar la gestión para lograr mayor } \\
\text { eficiencia en la protección focalizada } \\
\text { y especializada. Se deben crear } \\
\text { nuevas instituciones y mecanismos } \\
\text { de regulación, coordinación, gestión } \\
\text { eficiente del gasto, mejorando la } \\
\text { focalización. }\end{array}$ & $\begin{array}{l}\text { Crear una nueva institucionalidad de un } \\
\text { sistema de protección integral con un } \\
\text { órgano rector del sistema. Para ello es } \\
\text { esencial crear un sistema de garantías } \\
\text { de la niñez normativa acorde a la } \\
\text { convención. }\end{array}$ \\
\hline
\end{tabular}

Fuente: elaboración propia.

vs. el sistema de protección universal e integral. Esto bajo un primer nivel de marco interpretativo que desde el campo normativo establece la institucionalización de las políticas a la niñez bajo los parámetros de la Convención de Derechos del Niño como parte del marco maestro.

En el análisis sobre quiénes tienen voz en la discusión sobre la reforma, como organizaciones de la sociedad civil, del sector público, académicos, 
organismos internacionales y partidos políticos, se observa variabilidad en el posicionamiento de los actores en los marcos interpretativos. La identificación con marcos interpretativos de los partidos políticos con representación parlamentaria es relevante, pues la reforma se diseñó en torno a diversos proyectos de ley discutidos en el Congreso. Se establece que existen partidos que se expresan en un único marco interpretativo, como los partidos de la derecha (Unión Demócrata Independiente, Renovación Nacional y Amplitud), que se identifican con el marco interpretativo proteccionista, y los de la izquierda (Partido Comunista, Partido Socialista, Izquierda Ciudadana y Revolución Democrática), que se identifican más con el marco interpretativo de enfoque de derechos, pero expresan asimismo el conflicto de marcos interpretativos. Sin embargo, partidos como el Partido Demócrata Cristiano y el Partido por la Democracia, ambos de la coalición de centroizquierda gobernante de Bachelet, Nueva Mayoría, tienen presencia en más de un cuadrante, lo que significa una variabilidad amplia en sus concepciones, ya que se acercan a uno u otro marco interpretativo, lo que debilita la posición del gobierno en torno a reformas identificadas con el marco interpretativo de enfoque de derechos.

Por otra parte, la atribución de roles muestra la complejidad del problema abordado, pues, al ser multidimensional, sus causas están en la familia, el contexto de pobreza y cultura, así como en los déficits y la dispersión de políticas públicas o las concepciones de niñez, entre otras. Desde el análisis de marcos interpretativos, el proceso de reforma examinado se puede entender desde las posibilidades de agencia de los actores, donde sus valores, ideas e ideología, así como los contextos, son relevantes de considerar.

\subsection{Proceso de alineación a través de la amplificación y la extensión de los marcos interpretativos de las politicas}

Se puede observar, como parte de los resultados del análisis de codificación axial, un proceso de alineación entre el marco interpretativo secundario, sostenido en la reforma de gobierno, y el marco maestro de enfoque tutelar imperante, lo que permite establecer la categoría selectiva, mostrar como se integran actores y discursos mediante los procesos de alineación de marcos.

Los actores gubernamentales que impulsaban la reforma desde el enfoque de derechos cuestionaron el marco maestro y exigieron una reformulación agregando nuevos retos para fortalecer el rol del Estado como garante de derechos.

A partir del análisis axial, se establece que hay una amplificación del marco interpretativo secundario de enfoque de derechos, pues integra los marcos a través de un valor o creencia que permite una estrategia discursiva de difusión y legitimación, de manera que aumenta la cantidad de actores que se enfrentan al problema público de acuerdo con el enfoque de derechos y rol garante del Estado. En este sentido, la idea de que es necesaria una mayor integración en el sector con un órgano rector y el fortalecimiento de una institucionalidad basada en la garantía de los derechos de la niñez fue promovida por los actores durante la elaboración de la política pública entre 2014 y 2018. El proceso de 
la amplificación del marco de la política revela las decisiones de los actores que impulsan la reforma para superar la indiferencia de un tema que no ha sido tradicionalmente prioritario en las políticas públicas (como sí lo son los temas de economía, salud, vivienda o educación), para inspirar y movilizar apoyo para el cambio de políticas.

Se requiere, entonces, un cambio de enfoque y una nueva mirada en materia de protección de los derechos de la infancia y adolescencia, desde una perspectiva global de los derechos humanos, integral. No se trata solo de la situación irregular, sino también de la defensa de los primeros y de generar una cultura de respeto y protección de los menores de edad. (Boletín 10.314$06,15 / 03 / 17)$

En este proceso de alineación, destaca la capacidad de los actores (especialmente los gubernamentales) para volver a legitimar el rol del Estado en la intervención en materia de niñez. Hay una amplificación del discurso político del marco maestro, centrado en la efectividad del Estado en términos de mejorar su fiscalización, recursos y gestión, que incorpora la necesidad de crear nuevas instituciones, mecanismos y normas que fortalezcan y mejoren la acción estatal. Además, hay una amplificación del marco maestro al incorporar la noción de una política pública que no solo se refiera a la infancia con necesidades de protección especial, sino a todos los niños, niñas y adolescentes de manera universal, como plantea la convención.

Por otro lado, el proceso de amplificar ciertas creencias también implica la recolección de evidencia sobre la eficiencia de fortalecer un marco interpretativo incorporando a nuevos actores para que participen de la discusión, como las organizaciones de la sociedad civil. En términos de actores, el gobierno siguió siendo principalmente el creador de políticas y el ejecutor, pero el marco interpretativo maestro mantuvo la subsidiariedad y la asociación público-privada para la provisión de servicios de protección a la niñez.

El proceso de alineamiento de marcos implicó también la extensión de los límites del marco maestro. Los actores (y las organizaciones que conforman) reorganizaron sus prioridades incorporando valores e intereses de diversos grupos y diferentes a los que tenían inicialmente. En el caso de las políticas públicas hacia la niñez, los actores impulsores de la reforma asumieron los valores e intereses de los partidos políticos y representantes de las organizaciones de la sociedad civil, además de organismos internacionales, especialmente el Unicef, quienes participaron en la formulación de la reforma. Estos fueron los mismos grupos cuyos valores e intereses comenzaron a destacarse en el marco maestro, especialmente después de la crisis de legitimidad de la principal institución pública encargada de resguardar a niños y niñas vulnerados en sus derechos, el Servicio Nacional de Menores (Sename), fundado sobre las bases del enfoque tutelar, judicial y focalizado. Esto se produjo a raíz de casos de maltrato y muertes de niños bajo la protección del Estado en hogares de internamiento a los cuales fueron derivados por los tribunales. 
De manera que la crisis del Estado, expresada en las violaciones a los derechos de niños, niñas y adolescentes que el Estado debía resguardar, fue un factor que dio urgencia a la reforma. Por otro lado, se marcó como prioridad el respeto de los derechos de la infancia expresados en la CDN. Este proceso implicó la extensión del marco interpretativo maestro de las políticas, que integró una aceptación de los principios de la CDN, pero a la vez mantuvo, de manera paralela y contradictoria, las visiones del propio marco maestro, como el predominio del derecho preferente de los padres sobre la autonomía progresiva del niño, que es un principio base de la convención; el rol de Estado subsidiario y focalizado en la atención de la población más pobre, y el enfoque tutelar.

Este proceso de alineación por extensión de marcos llevó a modificaciones en la idea de niñez, de derechos de la infancia, de su participación y de la garantía de esos derechos.

En la categoría selectiva se puede identificar un proceso de alineación de marcos interpretativos de políticas, en el cual los actores de la política revalorizan algunos de sus propios puntos de vista sobre la niñez. De esta forma, la extensión del marco interpretativo secundario no rompe con la hegemonía del marco interpretativo maestro proteccionista y tutelar.

Si bien celebramos la creación de la Defensoría y de la Subsecretaría, ¿̨no creen que sería preferible contar previamente con un servicio de protección especializada, particularmente para ir en auxilio de esos miles de niños y niñas cuyo destino fatalmente los despojó de un entorno familiar que los contuviera? ¿Podemos seguir avanzando si todavía faltamos al compromiso, adquirido por el Estado de Chile décadas atrás, con la Convención sobre los Derechos del Niño en relación con tener una ley marco de los derechos de la infancia? En principio, podemos seguir adelante con este proyecto, pero hago presente al gobierno que falta el corazón de la agenda, y que sin él no se vislumbran mayores cambios a la infancia vulnerada, esa que más nos necesita y a la que le hemos fallado como Estado. (Diputada Marcela Sabat. Tramitación de la Subsecretaría de la Niñez [RN, derecha], 19/12/2017)

La investigación muestra que hay dos visiones de política pública expresadas en los marcos interpretativos que están tensionados, y que, dada la predominancia del marco interpretativo maestro, en el proceso de análisis de las categorías axiales se muestra que hubo modificaciones sin cambio de paradigma hacia un enfoque de derechos, que es lo que buscaba la reforma impulsada.

De manera que en la categoría selectiva se establece que el marco interpretativo secundario de enfoque de derechos logra introducir algunas de sus ideas centrales y generar consenso con el marco proteccionista. Muestra de ello es la aprobación de las leyes que crean la Subsecretaría de la Niñez (Ley 20.090) y la Defensoría de la Niñez (Ley 21.067). Sin embargo, la lógica central de este marco interpretativo, expresada en el proyecto que creaba un sistema de garantías de derechos de la niñez, no logró ganar terreno en la disputa simbólica, lo que se materializa en que este sistema de garantías de la niñez no consiguió 
aprobarse. Así, en ese proyecto, se generó la máxima expresión de divergencia de marcos interpretativos. El responsable de resolver el problema se estructura en torno a la atribución de rol del Estado como principal actor de cambios.

Los funcionarios de gobierno tuvieron capacidad para construir este marco interpretativo y lograr acuerdos principalmente con los actores políticos del Congreso. Aunque hubo diferencias entre los discursos políticos de los partidos, los ministros responsables y el Consejo de la Infancia en el énfasis de las políticas (referidas en particular al rol del Estado como garante de derechos y a la introducción de medidas administrativas, ambas con considerable aumento del presupuesto), el proceso de alineación incluyó cambios significativos realizados en la dirección de la reforma, de manera que dio preponderancia a los proyectos de cambio institucional y quitó prioridad al proyecto de ley de Sistema de Garantías de Derechos de la Niñez.

\section{Conclusiones}

La reforma de la política de infancia del gobierno de Bachelet (2014-2018) tuvo como objetivo crear una nueva institucionalidad pública, con nuevos organismos, mecanismos de coordinación interinstitucional y el fortalecimiento de las atribuciones de los organismos existentes, y se puso como eje principal velar por los derechos de niños, niñas y adolescentes contenidos en la CDN. Esta reforma fue considerada una tarea estratégica y logró resultados parciales.

El análisis muestra la relevancia de considerar los valores, las ideas y la ideología de los actores políticos para explicar las limitaciones, así como las oportunidades de cambio de políticas. No obstante, desde el análisis de marcos interpretativos realizado, se observa que los consensos iniciales entre los actores respecto a la importancia de la infancia y la necesidad de generar políticas públicas acordes a la Convención de Derechos del Niño derivaron en diferencias sustantivas que impidieron que la reforma lograra concretarse.

Asimismo, el análisis también permite observar que existe un cambio en las políticas sobre la infancia, pero que se mantiene el enfoque proteccionista y tutelar, donde el Estado, en vez de ejercer un rol garante de derechos, tiene un rol subsidiario. De manera que los derechos de los niños, las niñas y adolescentes, en especial los referidos a su participación y a su consideración como sujetos de derechos, no logran permear los cambios realizados.

El análisis, en su conjunto, muestra que en la formulación de política pública tiene un papel crucial la dimensión simbólica y valorativa, y resalta la preponderancia respecto a dimensiones de costos y beneficios, y dinámicas institucionales. Este es un aspecto de relevancia que muchas veces queda oscurecido en el análisis de política pública.

Las observaciones muestran la relevancia y la complejidad de las disputas y tensiones de significados y valores ante un problema público entre los policy makers. De manera que, dependiendo de los valores y significados desde donde se entiende el problema, se toman decisiones en términos de diagnóstico o modelo de solución (Hulst y Yanow, 2016; Verloo, 2005). 
Por lo tanto, el proceso de cambio de las políticas debe integrar y tener en cuenta la manera en que los actores participantes encuentran límites y posibilidades de significación y sentido, así como de evidencia, para que el conflicto de marcos interpretativos de las políticas se exprese (Verloo, 2005; Bustelo y Lombardo, 2006).

En la formulación de la reforma de la política pública de niñez y adolescencia en Chile, que buscaba el cambio hacia un sistema de protección con enfoque de derechos, se logró ampliar su condición de posibilidad al fortalecer la organización institucional en el campo de acción pública hacia la niñez con dos nuevos organismos: la Subsecretaría de la Niñez y la Defensoría de la Niñez. Sin embargo, la larga estela de políticas focalizadas, tutelares y asistencialistas, centradas en reducir factores de riesgo de la niñez en condición de pobreza, siguió dominando al mantener los principios del marco interpretativo maestro. De esta manera, la primacía de los factores de divergencia y conflicto terminan por imponerse como obstáculo a la reforma impulsada.

El conflicto entre ambos marcos está lejos de su fin. El gobierno actual de Sebastián Piñera (2018-2022), perteneciente a la coalición de derecha Chile Vamos, está llevando a cabo una agenda de cambios en materia de niñez y adolescencia, por lo que el escenario de transformación o de mantenimiento del statu quo está abierto. Así pues, serán necesarios nuevos análisis que extiendan y profundicen en los factores condicionantes implícitos y explícitos que definen las posibilidades y las limitaciones para el cambio de las políticas públicas de infancia en Chile.

Para ello son claves los procesos de alineación efectiva entre los frames de los diferentes actores (en este caso, entre los gubernamentales y políticos) y el marco maestro. Ello permitiría que los actores reacondicionen puntos de vista y reconstruyan diagnósticos, pronósticos y motivaciones para su acción en el ámbito de las políticas públicas de infancia. En este proceso, la discusión pública y la capacidad de agencia de los actores permitirían impulsar procesos de alineación de marcos interpretativos.

Finalmente, el estudio reportado muestra que, en la formulación de cambios en políticas públicas que abordan problemas complejos, como es el caso de la política hacia la infancia y adolescencia, el análisis de los marcos interpretativos y de los procesos de alineación de marcos permite ilustrar cómo el marco de creencias, significados y valoraciones juega un rol crucial para limitar o amplificar las condiciones y las posibilidades para el cambio de la política. Así, revela la potencialidad del análisis de valores, creencias e ideologías para entender y avanzar en la elaboración de reformas a políticas públicas en áreas de alta disputa y controversia como el caso analizado.

\section{Agradecimientos}

Este estudio ha sido desarrollado en el marco del Proyecto Fondecyt $\mathrm{N}^{\circ}$ 11.160.330, «Cambio en las políticas hacia la infancia y adolescencia en Chile. Análisis de los marcos interpretativos de instituciones y actores relacionados", del Consejo de Ciencia y Tecnología (Conicyt) de Chile. 


\section{Referencias bibliográficas}

BARBIERI, Nicolás (2015). «A narrative-interactionist approach to policy change analysis. Lessons from a case study of the cultural policy domain in Catalonia». Critical Policy Studies, 9 (4), 434-453. <https://doi.org/10.1080/19460171.2015.1014519>.

Bosomworth, Karyn (2015). "Climate Change Adaptation in Public Policy: Frames, Fire Management, and Frame Reflection». Environment and Planning C: Government and Policy 33(6), 1450-66. <https://doi.org/10.1177/0263774X15614138>.

Broad, Rose; Turnbull, Nick (2018). «From Human Trafficking to Modern Slavery: The Development of Anti-Trafficking Policy in the UK». European Journal on Criminal Policy and Research, 25, 119-133. <https://doi.org/10.1007/s10610-018-9375-4>.

Bedregal, Paula; Torres, Andrea; Carvallo, Camila (2014). "Chile Crece Contigo: El desafío de la protección social a la infancia». Documento de Trabajo. Santiago: Programa de las Naciones Unidas para el Desarrollo.

Benford, Robert; Snow, David (2000). «Framing processes and social movements: An overview and assessment». Annual Review of Sociology, 26, 611-639. $<$ https://doi.org/10.1146/annurev.soc.26.1.611>.

Bustelo, María; Lombardo, Emanuela (2006). "Los "marcos interpretativos" de las políticas de igualdad en Europa: conciliación, violencia y desigualdad de género en la política». Revista Española de Ciencia Política, 14, 117-140.

Bustelo Graffigna, Eduardo (2005). «Infancia en indefensión». Salud Colectiva, 1, 253-284.

Collins, Mary Elizabeth (2018). "Comparative Analysis of State Policymaking in Child Welfare: Explaining Policy Choices». Journal of Comparative Policy Analysis: Research and Practice, 20 (4), 370-386. <https://doi.org/10.1080/13876988.2017.1390867>.

Conlon, Catherine; Carney, Gemma; Timonen, Virpi; Scharf, Thomas (2015). "Emergent reconstruction" in grounded theory: Learning from team-based interview research». Qualitative Research, 15 (1), 39-56. $<$ https://doi.org/10.1177/1468794113495038>.

Duffy, Joe; Collins, Mary; Sook Hyun, Kim (2018). "Linking family engagement with a rights perspective: Macro factors influencing practice». European Journal of Social Work, 21 (1), 45-60. <https://doi.org/10.1080/13691457.2016.1255925>.

EArls, Felton (2011). "Children: form rights to citizenship". Annals of the American Academy of Polictical and Social Science, 633, 6-16. <https://doi.org/10.1177/0002716210383637>.

Fischer, Frank (2003). Reframing public policy. Discursive politics and deliberative practices. Nueva York: Oxford University Press. <https://doi.org/10.1093/019924264X.001.0001>.

FIsCHer, Frank; GOTTWEIs, Herbert (eds.) (2012). The argumentative turn revisited: Public policy as communicative practice. Durham: Duke University Press. <https://doi.org/10.1111/spol.12033>.

Gaitán, Laura (2010). «Ser niño en el siglo xxi». Cuadernos de Pedagogía, 407, 12-17.

Gilbert, Neil; Parton, Nigel; Skivenes, Marit (eds.) (2011). Child protection systems: International trends and orientations. Nueva York: Oxford University Press. <https://doi.org/10.1093/acprof:oso/9780199793358.003.0004>. 
Goffmann, Erving (1986). Frame Analysis. An essay on the organization of experience. Boston: Northeasten University Press.

Gómez-Mendoza, Miguel Ángel; Alzate-Piedrahíta, María Victoria (2014). «La infancia contemporánea». Revista Latinoamericana de Ciencias Sociales, Niñez y Juventud, 12 (1), 77-89.

Grau, Olga (2011). "Representaciones sociales de la infancia, discursos y prácticas». Politicas Públicas para la Infancia, 43-55.

Hajer, Maarten (2009). Authoritative Governance: Policy Making in the Age of Mediatization Oxford: Oxford University Press. <https://doi.org/10.1093/acprof:oso/9780199281671.001.0001>.

Harris, Marian (2014). Racial disproportionality in child welfare. Nueva York: Columbia University Press. <https://doi.org/10.1080/08841233.2014.941777>.

Herrera-Seda, Constanza; Aravena-Reyes, Andrea (2015). Imaginarios sociales de la infancia en la política social chilena (2001-2012)». Revista Latinoamericana de Ciencias Sociales, Niñez y Juventud, 1 (13), 71-84.

Hulst, Merlijn van; Yanow, Dvora (2016). "From policy "frames" to "framing": Theorizing a more dynamic, political approach». American Review of Public Administration, 46 (1), 92-112. <https://doi.org/10.1177/0275074014533142>.

James, Allison (2013). Socialising children. Londres: Palgrave Macmillan UK. <https://doi.org/10.1057/9781137317339>.

Larraín, Soledad (2011). «De objeto de protección a sujeto de derecho». Políticas Públicas para la Infancia, 91-98

Lombardo, Emanuela; Meier, Petra (2009). «Power and Gender: Policy Frames on Gender Inequality in Politics in the Netherlands and Spain». Journal of Women, Politics \& Policy, 30 (4), 357-380. <https://doi.org/10.1080/15544770903266632>.

LOMBARDO, Emanuela (2005). «Integrating or setting the agenda? Gender mainstreaming in the European constitution-making process». Social Politics: International Studies in Gender, State \& Society, 12(3), 412-432. <https://doi.org/10.1093/sp/jxi022>.

López Rodríguez, Silvia (2011). «¿Cuáles son los marcos interpretativos de la violencia de género en España? Un análisis constructivista». Revista Española de Ciencia Política, 25, 11-30.

Maldonado, Francisco (2014). «Estado y perspectivas de la reforma proyectada en Chile sobre el sistema de protección de menores de edad». Revista Lust et Praxis, 20 (2), 209-334.

Mayall, Berry (2000). "The sociology of childhood in relation to children's rights». The International Journal of Children's Rights, 8 (3), 243-259. $<$ https://doi.org/10.1163/15718180020494640>.

Morlachetti, Alejandro (2013). Sistemas nacionales de protección integral de la infancia: fundamentos jurídicos y estado de aplicación en América Latina y el Caribe. Santiago de Chile: CEPAL.

Ortiz, Ligia Galvis (2009). La Convención de los Derechos del Niño veinte años después». Revista Latinoamericana de Ciencias Sociales, Niñez y Juventud, 7 (2), 587-619.

Pösö, Tarja; Skivenes, Marit; Hestbaek, Anne-Dorthe (2014). «Child protection systems within the Danish, Finnish and Norwegian welfare states-time for a 
child centric approach?». European Journal of Social Work, 17 (4), 475-490. $<$ https://doi.org/10.1080/13691457.2013.829802>.

Rein, Martin; Schön, Donald (1996). «Frame-critical analysis and frame-reflective policy practice». Knowledge and Policy: The International Journal of Knowledge Transfer and Utilization, 9 (1), 85-104. <https://doi.org/10.1007/BF02832235>.

RoBerTs, Mark (2016). "Communication breakdown: Understanding the role of policy narratives in political conflict and consensus». Critical Policy Studies, 12 (1), 82-102. <https://doi.org/10.1080/19460171.2016.1230507>.

Roggeband, Conny (2010). «The Victim-Agent Dilemma: How Migrant Women’s Organizations in the Netherlands Deal with a Contradictory Policy Frame». Signs: Journal of Women in Culture and Society, 35 (4), 943-967. $<$ https://doi.org/10.1086/651050>.

Schmidt, Vivien; Radaelli, Claudio (2004). «Policy change and discourse in Europe: Conceptual and methodological issues». West European Politics, 27 (2), 183-210. <https://doi.org/10.1080/0140238042000214874>.

Schön, Donald; Rein, Martin (1994). Frame Reflection. Toward the Resolution of Intractable Policy Controversies. Nueva York: Basic Books.

Sepúlveda, Magdalena (2014). «De la retórica a la práctica: el enfoque de derechos en la protección social en América Latina». CEPAL, Serie Políticas Sociales, 189. <https://doi.org/10.2139/ssrn.2438720>.

Shim, Junseop; Chisung, Park; Wilding, Mark (2015). «Identifying policy frames through semantic network analysis: An examination of nuclear energy policy across six countries». Policy Sciences, 48 (1), 51-83. <https://doi.org/10.1007/s11077-015-9211-3>.

Snow, David; Rochford, E. Burke; Worden, Steven; Benford, Robert (1986). «Frame alignment processes, micromobilization, and movement participation». American Sociological Review, 51 (4), 464-481. <https://doi.org/10.2307/2095581>.

Steensland, Brian (2008). "Why Do Policy Frames Change? Actor-Idea Coevolution in Debates over Welfare Reform». Social Forces, 86 (3), 1027-1054.<www.jstor. org/stable/20430786>.

Stone, Deborah (2002). Policy Paradox: The Art of Political Decision Making. Tercera edición revisada. Nueva York: W. W. Norton.

Strauss, Anselm; Corbin, Juliet (2002). Bases de la investigación cualitativa. Técnicas y procedimientos para desarrollar la teoría fundamentada. Antioquia: Universidad de Antioquia.

Vergara del Solar, Ana (2014). «Public policy discourses during Chilean post-authoritarian democracy: A case of discursive colonization by the language of the free market». Childhood, 22 (4), 432-446. <https://doi.org/10.1177/0907568214549079>.

Verloo, Mieke (2005). «Mainstreaming Gender Equality in Europe. A Frame Analysis Approach». Greek Review of Social Research, 117, 11-34. <https://doi.org/10.12681/grsr.9555>.

Villalta, Carla; Llobet, Valeria (2015). «Resignificando la protección. Los sistemas de protección de derechos de niños y niñas en Argentina». Revista Latinoamericana de Ciencias Sociales, Niñez y Juventud, 1 (13), 167-180.

YANow, Dvora (2000). Conducting interpretive policy analysis. Newbury Park, California: Sage. 